\title{
Design and Implementation of Wireless Based Android Controlled Spy-vehicle
}

\author{
Nyi Nyi Tun ${ }^{1}$, Kyaw Thiha ${ }^{2}$ \\ ${ }^{I}$ (Mechatronic Engineering, Mandalay Technological University, Myanmar) \\ ${ }_{2}^{2}$ (Mechatronic Engineering, Mandalay Technological University, Myanmar)
}

\begin{abstract}
A new version of wireless controlled vehicle is proposed for spying purposes. This research will apply the smart phone to transmit bluetooth wireless data to the Arduino-Uno then it will give the data for vehicle's movement. This robot supposes a movable spy robot with wireless system by using Arduino-Uno as a main controller. The spy robot is made up of an IP camera, DC motors, servo motors for camera rotation, lithium rechargeable ion batteries, and four movable wheels. Smart phone camera is used as an IP camera to capture livestreaming video surrounding the robot and that information will be appeared in user's smart phone GUI window. In this vehicle, the bluetooth signals transmitted from the user's smartphone are used for vehicle motion and then the camera mounted on robot will take the video livestreaming. This livestreaming information is sent back to the user's smart phone via wifi wireless system. For the system, the simulation result is done by using Proteus Professional schematic software. Arduino programming language is used for Arduino-Uno and Basic4 Android programming is used to write desired App in smart phone.
\end{abstract}

Keywords : Basic4 Android Programming, Bluetooth Wireless System, DC motor, Spy-vehicle, Wifi Wireless System

\section{Introduction}

Nowadays spy-vehicles have been widely used in various kinds of fields like industries, academic, research and development, militaries and so on. The spy-vehicles are small vehicles designed for spying, surveillance and inspection purposes. They can be customized for specific applications and are made with some special features. They are remotely controlled vehicle, equipped with a camera, transmitting video data to the intervention troop. Troopers can access the situation in the room where the small vehicle is thrown. They are made to be small and compact enough to easily transport. Most of them are designed for use in rough terrain. In brief, spy vehicles must be small and lightweight, robust, mobile, tele-operated (wireless) [1]. In this research, a movable spy-vehicle with a smartphone controller is implemented. The vehicle is not quite huge one and designed to be easy in transportation. The remote controller, smartphone is to control the robot to reach the desirable destination. The spy-vehicle is fundamentally made up of smartphone, Arduino-Uno microcontroller, L298 motor driver, DC motors and four movable wheels. When the user drives the robot with bluetooth wireless technique via written App, the spy-vehicle will move to desired destination and spies video around the vehicle. The vehicle will transmit the video livestream that are spied around the vehicle and retransmit to the driver's smartphone via wifi wireless technique for a good spying system. By watching the video, the user will know the required information. The overall block diagram of the wireless based spy-vehicle is shown in Fig. 1.

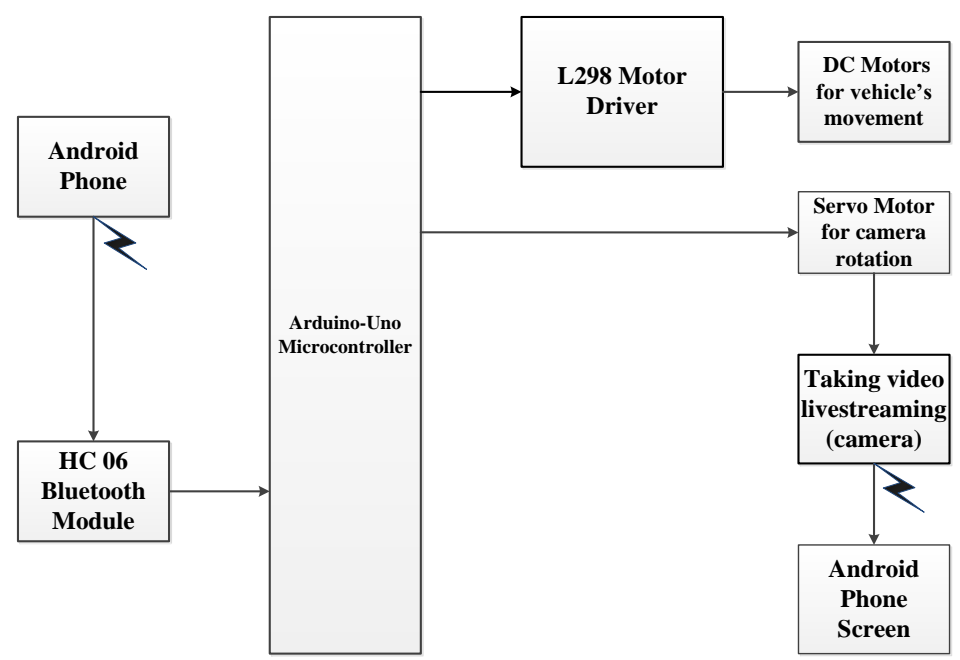

Fig. 1. Overall Block Diagram of the System 


\section{Hardware Configuration}

In this paper, the system is mainly composed of Arduino-Uno microcontroller, L298 motor driver, four robot wheels and dc motors, servo motors, HC06 bluetooth module, two smartphones and boost converters respectively.

\section{Modeling a de motor}

DC motors are widely used in robotics because of their small size and high energy output. The key characteristics of DC motors are high speed, low torque and its reversibility. The spy robot uses four 3V 6V DC motors as shown in Fig. 2. This DC motor is dual axis gear motor, which reduction ratio is 1:48. Working voltage of this motor is $3 \mathrm{~V}$ to $6 \mathrm{~V}$. The unload current is $200 \mathrm{~mA}$ at $6 \mathrm{~V}$ and $150 \mathrm{~mA}$ at $3 \mathrm{~V}$ respectively. Motor's unload speed is $200 \pm 10 \% \mathrm{RPM}$ at $6 \mathrm{~V}$ and $90 \pm \mathrm{RPM}$ at $3 \mathrm{~V}$.

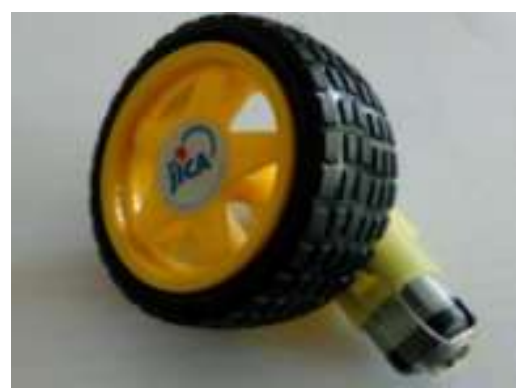

Fig. 2. DC motor and Wheel

To be modeling a DC Motor, simple circuit of its electrical diagram as shown in Fig. 3 is considered. To be modeling and simulate the DC motor, the following steps are to be made step by step;

Step1: Represent the DC motor circuit diagram

Step2: Represent system equations

Step3: Calculate the Transfer function

Step4: Convert to model block

\subsection{Closed-loop System consideration}

To perform the simulation of the system, an appropriate model needs to be established. Therefore, a model based on the motor specifications needs to be obtained. Fig. 3 shows the DC motor circuit with Torque and Rotor Angle consideration.

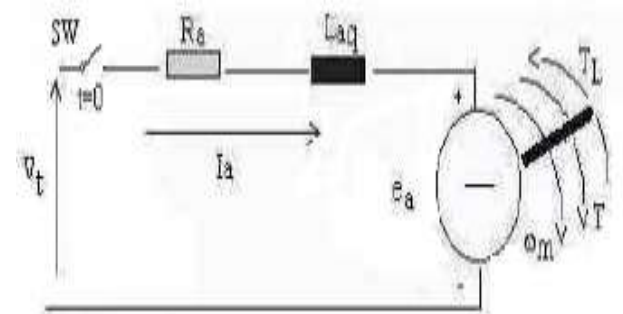

Fig. 3. Schematic Diagram of a DC Motor [2]

\subsection{System Equation}

The motor torque $\mathbf{T}$ is related to the armature current, $\mathbf{i}$, by a torque constant $\mathbf{K}$;

$$
T=K i
$$

The generated voltage, $\mathbf{e}_{\mathrm{a}}$, is relative to angular velocity by;

$$
e_{a}=K \omega_{m}=K \frac{d \theta}{d t}
$$

From Fig. 3 we can write the following equations based on the Newton's law combined with the Kirchoff's law:

$$
\begin{gathered}
J \frac{d^{2} \theta}{d t^{2}}+b \frac{d \theta}{d t}=K i \\
L \frac{d i}{d t}+R i=V-K \frac{d \theta}{d t}
\end{gathered}
$$




\subsection{Transfer Function}

Using the Laplace transform, equations (3) and (4) can be written as:

$$
\begin{gathered}
J s^{2} \theta(s)+b s \theta(s)=K I(s) \\
L s I(s)+R I(s)=V(s)-K s \theta(s)
\end{gathered}
$$

where $s$ denotes the Laplace operator. From (6) we can express $\mathbf{I}(s)$ :

$$
I(s)=\frac{[V(s)-K s \theta(s)]}{(R+L s)}
$$

and substitute it in (5) to obtain:

$$
J^{2} \theta+b s \theta(s)=\frac{K(V(s)-K s \theta(s))}{R+L s}
$$

This equation for the DC motor is shown in the block diagram in Fig. 4 From equation (8), the transfer function from the input voltage, $\mathbf{V}(s)$, to the output angle, $\square$, directly follows:

$$
G a(s)=\frac{\theta(s)}{V(s)}=\frac{K}{\left\{s\left[(R+L s)(J s+b)+K^{2}\right]\right\}}
$$

From the block diagram in Fig. 4, it is easy to see that the transfer function from the input voltage, $\mathbf{V}(s)$, to the angular velocity, $\boldsymbol{\omega}$, is:

$$
G v(s)=\frac{\omega(s)}{V(s)}=\frac{K}{\left\{\left[(R+L s)(J s+b)+K^{2}\right]\right\}}
$$

The motor specification of DC Motor which will be used in Spy-vehicle as shown in below.

Power $\mathrm{P}=1.2$ watts, Speed $\mathrm{N}=200 \mathrm{rpm}$ (max), $_{\text {, }}$ rotor inertia $\mathrm{J}$ is assumed to be 0.01 and Supply voltage $\mathrm{Vt}=6$ volts. Therefore for the max speed rpm of 200 , it can be calculate the torque constant $\mathrm{K}$;

$$
\begin{aligned}
\omega_{m} & =\frac{V t}{K}=\frac{(2 \pi N)}{60} \\
\mathrm{~K} & =0.2857 \text { and } \omega_{\mathrm{m}}=21 \mathrm{radsec}^{-1}
\end{aligned}
$$

By using equation 3 , for $\omega=\frac{d \theta}{d t}$

$$
K \times i=\frac{d \omega}{d t}+b \omega
$$

At the steady state (used as analyzed data), both $\mathbf{I}$ and $\boldsymbol{\omega}$ are stabilized;

$$
\frac{d \omega}{d t}=0
$$

Therefore, the total equivalent damping $\mathbf{b}$ can be chosen the value of;

$(0.2857 * 0.2)-\mathrm{b}(21)=0$

$$
\mathrm{b}=0.0027
$$

By calculating and assuming the require data as above, the following value are assigned to be used for our desire DC Motor Model.

$\mathrm{Vt}=6 \mathrm{~V} ; \mathrm{J}=0.01 ; \mathrm{b}=0.0027 ; \mathrm{K}=0.2857 ; \mathrm{R}=1.617$ ohms and $\mathrm{L}=0.001 \mathrm{H}$;

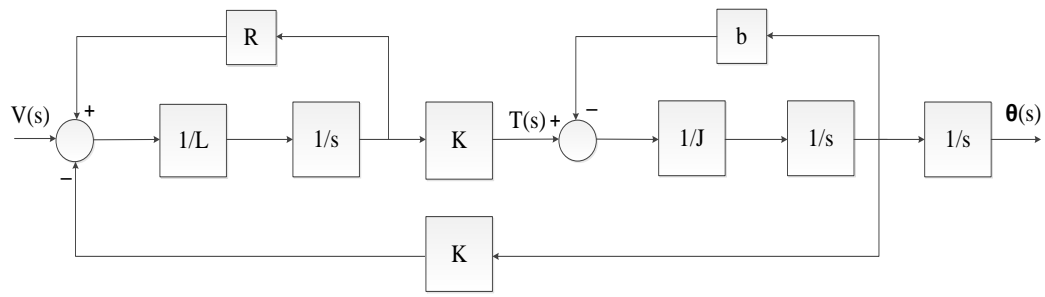

Fig. 4. A Block Model System of DC motor

In this research, the spy-vehicle is implemented by using two wireless systems such as bluetooth and wifi. Bluetooth data via smart phone is used for the vehicle movement and wifi is applied for the livestreaming video from spy-vehicle. Internet Protocol (IP) address is used to get the livestream on reality. And the required App is invented by using the Basic4 Android programming language. Thus, this section will explain the two wireless systems, IP address, basic4 Android App written method and livestreaming video. 


\section{Bluetooth wireless technology $(B W T)$}

Bluetooth wireless technology is a short range radio communication system designed for communication of devices like mobile phones, PDAs, notebooks, PCs, printers and headsets. Bluetooth technology operates in the unlicensed Industrial, Scientific and Medical (ISM) band at $2.420-2.485 \mathrm{GHz}$, using a spread spectrum, frequency hopping, full-duplex signal at a nominal rate of 1600 hops $\sec ^{\square 1}$ [3]. Bluetooth technology's Adaptive Frequency Hopping (AFH) capability was designed to reduce interference between wireless technologies sharing the $2.4 \mathrm{GHz}$ spectrum. AFH works within the spectrum to take advantage of the available frequency. This is done by detecting other devices in the spectrum and avoiding the frequencies they are using. This adaptive hopping allows for more efficient transmission within the spectrum, providing users with greater performance. The signal hops among 79 frequencies at $1 \mathrm{MHz}$ intervals to give a high degree of interference immunity. The heart of the Bluetooth specification is the Bluetooth protocol stack. By providing well layers of functionality, the Bluetooth specification ensures interoperability of Bluetooth devices and encourages adoption of Bluetooth technology. There are three classes of BWT radio devices, each with a different maximum range Class 1 (100 meters), Class 2 (50 meters) and Class 3 (10 meters) respectively. In this research, Class 3 is used for testing.

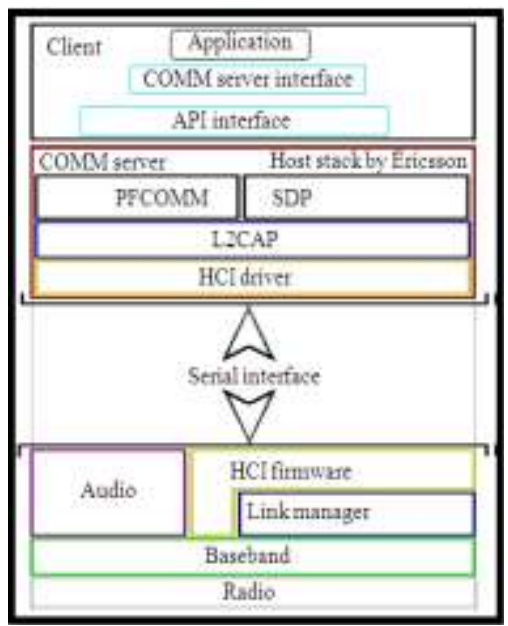

Fig. 5. Bluetooth protocol stack [4]

\section{Wireless fidelity}

WiFi is a universal wireless networking technology that utilizes radio frequencies to transfer data. WiFi allows high-speed internet connections without the use of cables. The term WiFi is a contraction of "wireless fidelity" and is commonly used to refer to wireless networking technology [5]. It is almost 10 times faster than a regular dial-up connection. It is also known as 802.11 networking and wireless networking. The 802.11 standard is defined through several specifications of WLANs. It defines an over-the-air interface between a wireless client and a base station or between two wireless clients. There are several specifications in the 802.11 family such as $802.11 \mathrm{a}, 802.11 \mathrm{~b}$ and $802.11 \mathrm{~g}$. The standard 802.11 pertains to wireless LANs and provides 1or 2-Mbps transmission in the 2.4-GHz band using either frequency-hopping spread spectrum (FHSS) or directsequence spread spectrum (DSSS). The 802.11a is an extension to 802.11 that pertains to wireless LANs and goes as fast as $54 \mathrm{Mbps}$ in the 5-GHz band. The 802.11a employs the orthogonal frequency division multiplexing (OFDM).The $802.11 \mathrm{~b}$ is an extension to 802.11 that pertains to wireless LANs and yields a connection as fast as $11 \mathrm{Mbps}$ transmission in the $2.4-\mathrm{GHz}$ band. The $802.11 \mathrm{~b}$ specification uses only DSSS. The 802.11g pertains to wireless LANs and provides $20+$ Mbps in the 2.4-GHz band. WiFi networks operate in the unlicensed 2.4 radio bands, with an $11 \mathrm{Mbps}(802.11 \mathrm{~b})$ or $54 \mathrm{Mbps}$ (802.11a) data rate, respectively [6]. The big advantage of WiFi is its simplicity. The higher frequency allows higher data rates. WiFi radios use much more efficient coding techniques that also contribute to the much higher data rates. For $802.11 \mathrm{a}$ and $802.11 \mathrm{~g}$, the technique is known as orthogonal frequency-division multiplexing (OFDM). For $802.11 \mathrm{~b}$, it is called Complementary Code Keying (CCK). The advantage of frequency hopping is that it is much more immune to interference.

\section{Internet Protocol (IP) Address}

An IP address is a numerical label assigned to each device participating in a computer network that uses the Internet Protocol for communication [7]. In this spy-vehicle, the livestream video is taken via android camera. It is used as an IP camera.To get the livestream video and information, the IP address of the camera is essential. IP address of android camera can be known via Droid IP camera App. Thus Droid IP camera App 
must be installed in the spy-vehicle's android camera. This IP address is encoded in basic4android program. The required App to drive the spy robot is written with B4A programming languages. The internet protocols consist of a suite of communication protocols, of which the two best known are the Transmission Control Protocol (TCP) and the Internet Protocol (IP). The Internet protocol suite not only includes lower-layer protocols (such as TCP and IP), but it also specifies common applications such as electronic mail, terminal emulation, and file transfer. An IP address is an identifier for a computer or device on a TCP/IP network. Each host on a TCP/IP network is assigned a unique 32-bit logical address that is divided into two main parts: the network number and the host number. The network number identifies a network and must be assigned by the Internet Network Information Center (Inter NIC). The host number identifies a host on a network and is assigned by the local network administrator. The 32-bit IP address is grouped eight bits at a time, separated by dots, and represented in decimal format (known as dotted decimal notation). Each bit in the octet has a binary weight $(128,64,32,16$, $8,4,2,1)$. The minimum value for an octet is 0 , and the maximum value for an octet is 255 . Fig. 6 illustrates the basic format of an IP address. IP addressing supports five different address classes: A, B, C, D, and E. Only classes A, B, and C are available for commercial use [8]. In this spy-vehicle, class C IP address is applied.

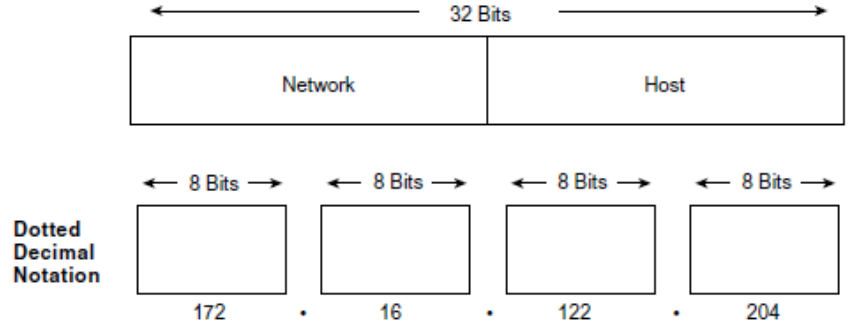

Fig. 6. An IP address consists of 32 bits, grouped into four octets [8]

\begin{tabular}{|c|c|c|}
\hline $\begin{array}{c}\text { Address } \\
\text { Class }\end{array}$ & $\begin{array}{c}\text { First Octet } \\
\text { in Decimal }\end{array}$ & $\begin{array}{c}\text { High-Order } \\
\text { Bits }\end{array}$ \\
\hline Class A & $1 \boxminus 126$ & 0 \\
Class B & $128 \boxminus 191$ & 10 \\
Class C & $192 \boxminus 223$ & 110 \\
Class D & $224 \boxminus 239$ & 1110 \\
Class E & $240 \oplus 254$ & 1111 \\
\hline
\end{tabular}

Fig. 7. A range of possible values exists for the first octet of each address class [8]

\section{Basic4 Android Programming}

Basic4Android is a rapid application development tool for native Android applications, development and marketed by anywhere Software Ltd. B4A is an alternative to programming with Java and the Android SDK. It is mainly operates in Microsoft Windows [9]. Basic4android depends on two additional (free) components such as Java JDK and Android SDK. When the Basic4Android application is started to write, the important software to install are Java JDK, Android SDK and Basic4 Android software on the computer. This programming can be used not only on 32-bit but also on 64-bit operating system. Then, it is needed to create Android Virtual Device (AVD) or emulator. Emulator is a software that simulate like real device on computer. Each Basic4 Android program runs in its own process. A process has one main thread which is also named the UI thread which lives as long as the process lives. A process can also have more threads which are useful for background tasks. A process starts when the user launches the application, assuming that it is not running already in the background. A Basic4 Android application is made of one or more activities, as in [10]. When a new activity is created, it will start with the following code;

The Basic Program without any additional code:

Sub Process_Globals

'These global variables will be declared once when the application starts.

'These variables can be accessed from all modules.

End Sub

Sub Globals

'These global variables will be redeclared each time the activity is created.

\begin{tabular}{llr}
\hline DOI: $10.9790 / 1676-110502178186$ & www.iosrjournals.org & $182 \mid$ Page
\end{tabular}


'These variables can only be accessed from this module.

End Sub

Sub Activity_Create (FirstTime As Boolean)

'Do not forget to load the layout file created with the visual designer.

'Activity.LoadLayout("Layout1")

End Sub

Sub Activity_Resume

End Sub

Sub Activity_Pause (UserClosed As Boolean)

End Sub

In the B4A programming code, variables can be either global or local. Local variables are variables that are declared inside a sub other than Process_Globals or Globals. Global variables can be accessed from all subs. There are two types of global variables. They are process variables and activity variables. Process variables are the variables that live as long as the process lives. These variables must declare inside sub Process_Globals and this sub is called once when the process starts. Activity variables are contained by the activity. These variables must declare inside Sub Globals.

\section{Video livestreaming}

Video streaming over wireless networks is compelling for many applications, ranging from home entertainment to surveillance to search-and-rescue operations. Interesting technical challenges arise when the unpredictable nature of the wireless radio channel meets the requirements of high data rate and low latency for video transport. Video streaming over wireless networks is compelling for many applications, and an increasing number of systems are being deployed. Video streaming of news and entertainment clips to mobile phones is now widely available [11]. In this research, video livestreaming was accomplished by making use of wifi Direct from spy-vehicle's camera to driver's phone. Wifi direct was used to maintain the goal of the vehicle being mobile and able to be used in any location and situation. By using wifi system, it allows connections between two mobile devices without the availability of a router. When compares to bluetooth, Wifi Direct surpasses it in both speed and range with speeds of up to $250 \mathrm{Mbps}$, which was a perfect fit for video streaming purposes. Wifi allows the high-speed connections without the use of cables. Instead of having router as an access point to communicate between two devices, Wifi direct allows a device to take the role of the access point. Fig. 8 is the B4A programmed GUI design for the research.

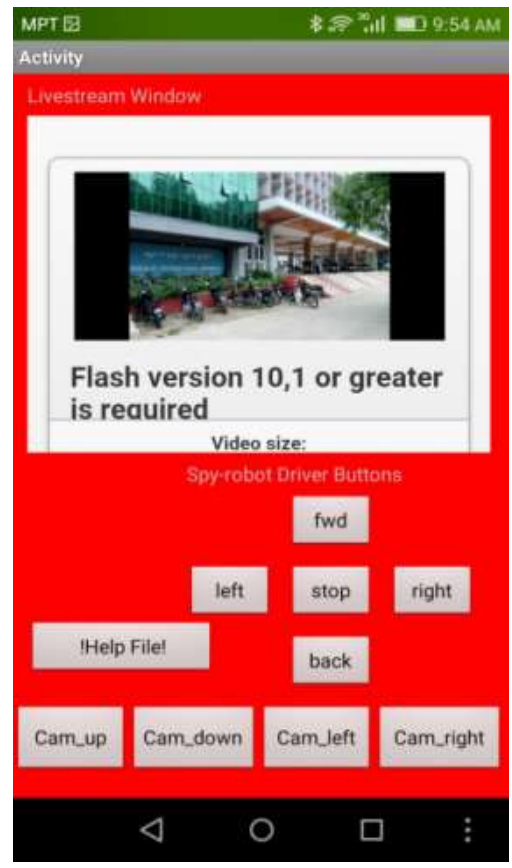

Fig. 8. B4A programmed GUI design for the spy-vehicle

In B4A GUI, livestream window on the top is to watch the vehicle where it arrives, what does camera take and to know the required information. There are five buttons to drive the robot in forward and backward directions, to turn left and right and completely stop. The button on the left corner is the help file for the users to 
direct how to use the App for spy-vehicle. There are four bottoms for vehicle's camera rotation such as camera up, camera down, camera left and camera right. This B4A App will enhance the spy-vehicle other than the analog remote control using RF modules. And it can lead to more comfortable and easy to control the robot just having a smartphone.

\section{Operation of the system}

In this research, the main controller is the Arduino-Uno that is given the data via HC06 Bluetooth module from user's android phone. The motors are driven via L298 motor driver. Servo motor is rotated for spy-vehicle's camera. Then, the information that is taken by camera will sent back to the user's phone screen for video livestreaming. The circuit diagram of spy-vehicle is shown in Fig. 9.The spy-vehicle is made up of Arduino-Uno microcontroller, L298 motor Driver, four robot Wheels and DC motors, HC06 Bluetooth Module and smartphone camera. This camera is used as an IP camera. In this vehicle, the Arduino-Uno acts as a main controller.

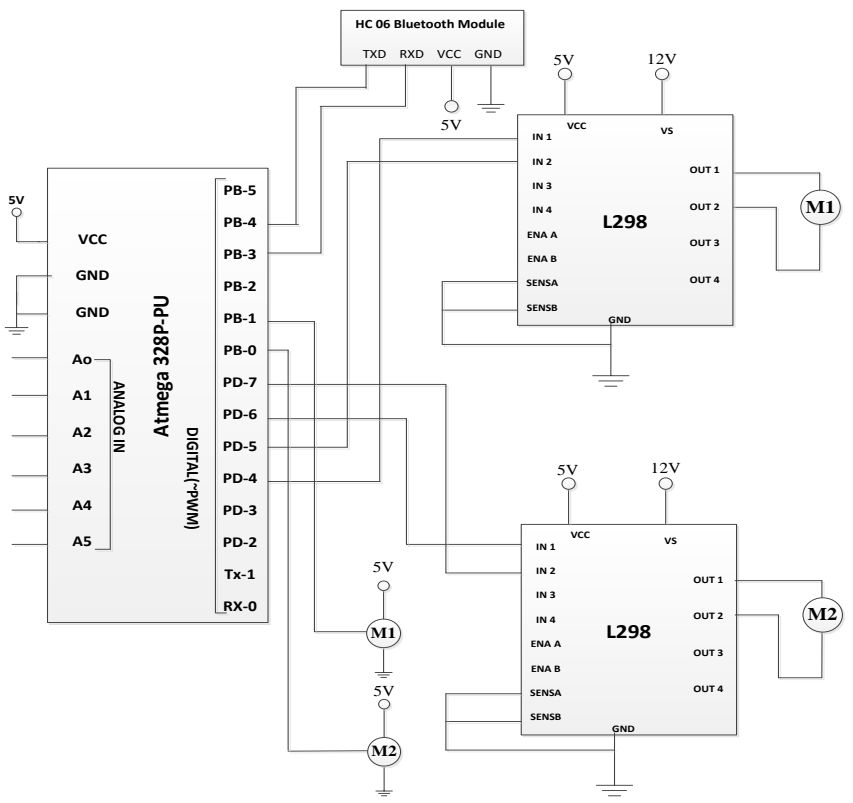

Fig. 9. Circuit diagram of the spy-vehicle

Each of the IN1, IN2 pin of L298 motor driver are connected by Arduino-Uno pin 4,5,6 and 7 respectively. The servo motors are connected by pin 8 and pin 9 of the Arduino-Uno. The bluetooth module is connected by pin 11 and pin 12 of Arduino for receiving and transmitting the data. This vehicle can move forward, backward, stop, left and right directions. The smartphone camera will rotate in four directions. The users can see the view about the surrounding conditions of the spy-vehicle in livestreaming window. Fig. 10 is the hardware result of the spy-vehicle.

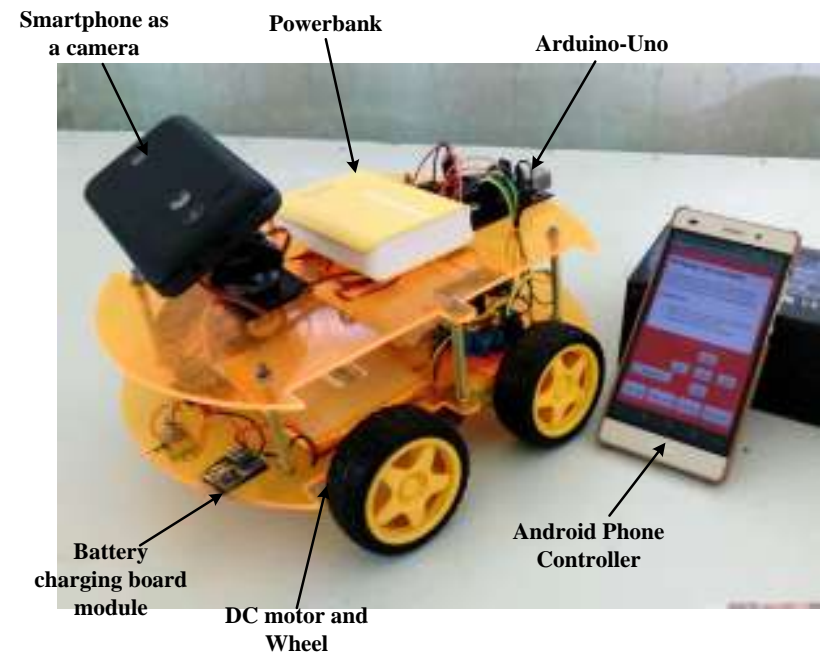

Fig. 10. Hardware result of the spy-vehicle 


\section{Spy-vehicle App Software}

In this paper, the smartphone App is implemented to use as the main driver of vehicle. App is written by Basic4n Android Programming language. Basic4 Android is the programming that is flexible for both android phones and tablets. Thus, it can be applied in our daily lives. In Fig. 11(a), the required App software is shown. The icon is named "Spy-vehicle" as it is aimed for spying purposes. In Fig. 11(b), the page is designed to describe the developer's university, department, thesis's title, and his name. Fig.11(c) is the help file view. In help file, the user will easily know how to use the App software by the given instructions. This help file can easily help the user to use spy-vehicle in reliability. Fig. 11(d) is the design of the warning message whether to quit or not. In addition, there are five buttons to control the spy-vehicle's direction. These buttons are designed as spy-robot diver buttons in B4A GUI design. The four buttons for camera rotation are also present. The final Basic4Android GUI design is shown in Fig. 8.

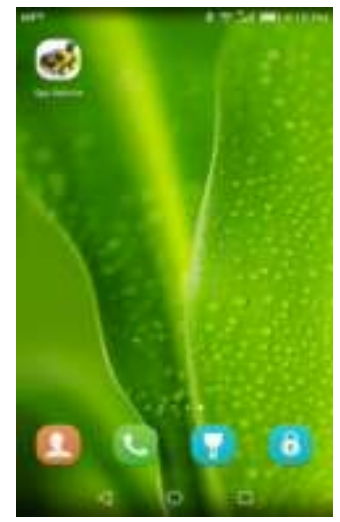

(a)

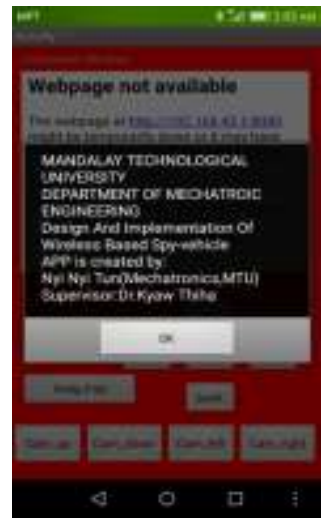

(b)

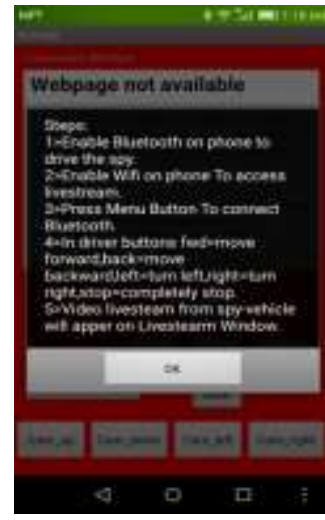

(c)

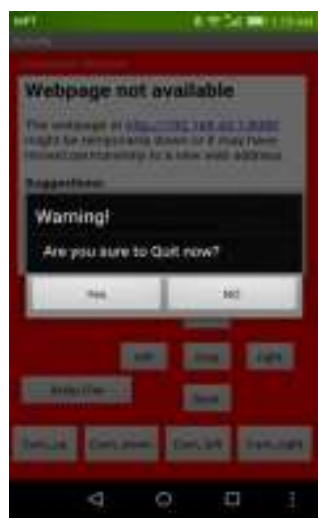

(d)

Fig. 11. (a) Icon of the spy-vehicle on smartphone (b) Design of the developer profile (c) Design of the Help

File in Spy-vehicle (d) Dialogue box for warning to quit

\section{Simulation test and result}

In this paper, the simulation and test result are divided by two sections. The first section is done for spy-vehicle's motors and servo motors testing. And, the second section is done for video livestreaming testing. The simulation result is done by using Proteus professional schematic software and. After the Arduino program is compiled as hex file, the compiled code is sent to the ATMega328P-Pu in Proteus diagram. In Proteus Simulation, the two DC motors are connected by output pins of the L298 motor drivers. The servo motors are connected with the pin 8 and 9 of Arduino. The two DC motors will rotate in four directions and stop. The servo motors will rotate within the range of -90 to +90 degree. The simulation result is shown in Fig. 12. The second section is tested for livestream testing. The spy-vehicle is made up of a smartphone camera that give out of IP address and it operates with wifi wireless system. The user will know the information by watching the livestream video in livestream window in user's smartphone. For the whole system, the spy-vehicle is not quite huge one and designed to be easy in transportation.
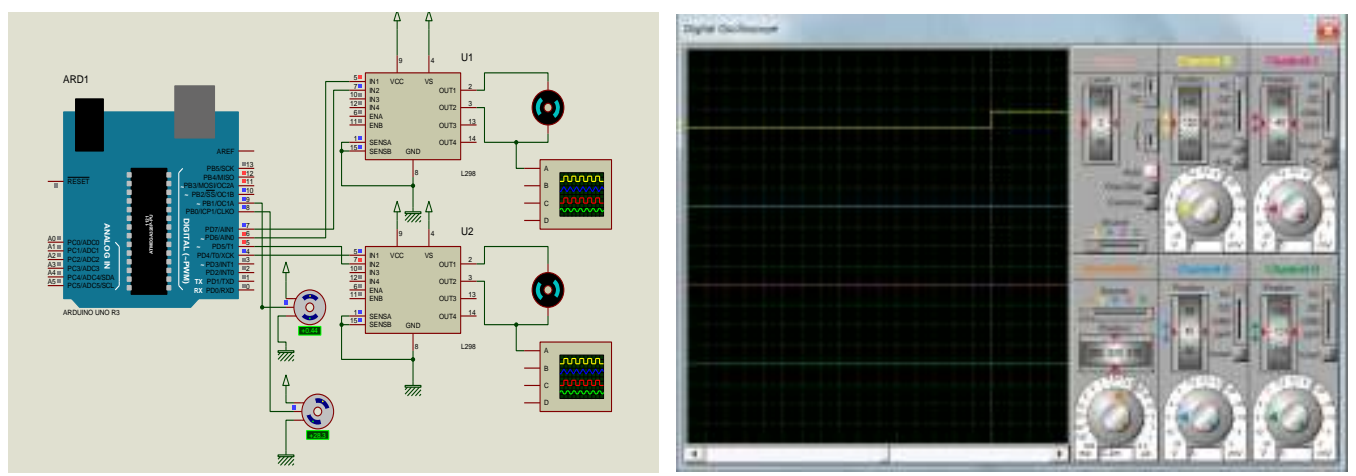

Fig. 12. Proteus Simulation of the DC motors and output waveforms

\section{Conclusion}

In conclusion, this paper describes wireless based spy-vehicle. In this research, two wireless systems are bluetooth and wifi system. This wireless systems are better than wireless RF communication. As BWTenabled devices operate in the unrestricted 2.4 - Gigahertz and wifi system can operate more than100 feet, this 
research can overcome the wireless limitation range rather than RF radio signal. Moreover, this research is aimed for using the smartphone to drive robot and to watch livestream. So, it is not necessary to use PC, Capture Card or TV Tuner Card to watch livestream video and images. Thus, the cost of this robot is inexpensive and suitable for research and scientific works.

\section{Acknowledgements}

The author is very thankful to Dr. Myint Thein, Rector of Mandalay Technological University, for his encouragement, invaluable permission and his kind support in carrying out this paper work. The author is deeply grateful to Dr. Wut Yi Win, Associate Professor and Head, Department of Mechatronic Engineering, Mandalay Technological University for supplying all necessary things. The author also wishes to thank to supervisor Dr. Kyaw Thiha, Associate Professor, Department of Mechatronic Engineering, Mandalay Technological University, accomplished guidance, his willingness to share his ideas and, helpful suggestions and for his patience, continuous supervision and encouragement during a long period of this paper. The author would like to express his indebtedness and deep gratitude to his beloved parents, for their kind support and understanding during the whole course. With this paper, the author has to hold one in honours to all teachers teaching from kindergarten to University. The author is deeply thankful to Japan International Cooperation Agency (JICA) for their support the required funds and necessary equipment during the research period.

\section{References}

[1]. Design and Implementation of Remote Operated SpyRobot Control System by Wai Mo Mo Khaing, Department of Mechatronic EngineERING, MANDALAy TECHNOLOGICAL University.

[2]. Analysis on Modeling and Simulink of DC Motor and Its Driving System Used for Wheeled Mobile Robot by Wai Phyo Aung, Mandalay Technological University

[3]. Bluetooth wireless Technology basic, Copyright 2004 Hewlett Packkard Development Company, L.p.

[4]. Bluetooth Wireless Monitoring, Managing and Control forInter Vehicle in Vehicular Ad-Hoc Networks by Helia Mamdouhi, Sabira Khatun and Javad Zarrin Department of Computer and Communication System Engineering, Faculty of Engineering, University Putra Malaysia, Malaysia

[5]. How wifi works by Marshell Brain pdf

[6]. Wifi Wireless SystemCommunication, tutorialspoint, www.tutorialspoint.com

[7]. What is IP Address :www.ip address+pdf.com

[8]. Internet Protocols(Chapter(30))+pdf

[9]. Basic4Android Step by step guide for your first Android Programming + pdf

[10]. Basic4AndroidTutorials;http://www.basic4Android.com

[11]. Android Controlled Spy-Robot by Prof.Y. M. Naik, Chiranjivi, M.Despande, Ravija.R.Shah, Rashmi.R. Kulkarni, Department of Computer Science and Engineering

[12]. Bluetooth Operated Robot Vehicle Using Mobile Android App by Devesha Parmar, Devashish Tripathi, Agam Sahni, Pankaj Singh; Department of Electronics and Communication Engineering, SRM University

[13]. War field Robot Controlled By Android Phone by Prof. Jagdish Patel, Akshay Malik,Vrushali Thakare, Rahul Rajput 